Revue d'histoire de l'enfance « irrégulière »

Le Temps de l'histoire

$14 \mid 2012$

Enfances déplacées. (I) en situation coloniale

\title{
Les « rapatriements » en France des enfants eurasiens de l'ex-Indochine
}

Pratiques, débats, mémoires

The "repatriation" to France of Eurasian children from former Indochina:

Practices, debates, memories

\section{Yves Denéchère}

\section{OpenEdition}

Journals

Édition électronique

URL : http://journals.openedition.org/rhei/3398

DOI : 10.4000/rhei.3398

ISSN : $1777-540 \mathrm{X}$

Éditeur

Presses universitaires de Rennes

\section{Édition imprimée}

Date de publication : 30 décembre 2012

Pagination : 123-141

ISBN : 978-2-7535-2194-0

ISSN : $1287-2431$

Référence électronique

Yves Denéchère, «Les « rapatriements » en France des enfants eurasiens de l'ex-Indochine », Revue d'histoire de l'enfance « irrégulière » [En ligne], 14 | 2012, mis en ligne le 30 décembre 2014, consulté le 23 avril 2019. URL : http://journals.openedition.org/rhei/3398; DOI : 10.4000/rhei.3398

Ce document a été généré automatiquement le 23 avril 2019

(c) PUR 


\title{
Les « rapatriements » en France des enfants eurasiens de l'ex-Indochine
}

\author{
Pratiques, débats, mémoires \\ The "repatriation" to France of Eurasian children from former Indochina: \\ Practices, debates, memories
}

Yves Denéchère

\section{Introduction}

1 Deux films documentaires réalisés en 2009 et 2010, et diffusés par la télévision publique, ont mis en lumière le sort des enfants eurasiens nés à la fin de l'Indochine coloniale de pères français et de mères indigènes ${ }^{1}$. Quelques années plus tôt, un livre de l'historienne Emmanuelle Saada avait défini avec précision les enjeux de la question du métissage dans les colonies en analysant la biopolitique suivie à cet égard ${ }^{2}$. En effet, après la fin de la guerre d'Indochine (1946-1954) la Fédération des œuvres de l'enfance française d'Indochine (FOEFI) envoya en France des centaines d'enfants eurasiens. Cet épisode n'est pas sans rappeler la récupération des enfants nés après 1945 de pères soldats français et de mères allemandes dans la zone française d'occupation (ZFO) en Allemagne sur fond de populationnisme d'État bien défini par Paul-André Rosental ${ }^{3}$. Dans un cas comme dans l'autre, la dimension biopolitique de l'entreprise migratoire contrainte est manifeste. Des bébés, des enfants, des adolescents sont devenus des objets de politiques voulues par les autorités françaises et mises en œuvre par des organisations diverses. Des mères ont été séparées de leurs enfants; des pères - qui dans l'immense majorité des cas n'avaient pas reconnu ces enfants - sont restés ignorants de leur sort et de celui de leurs mères. Quand en 1979 - époque où il définit la biopolitique - Michel Foucault voit dans les boat people " un présage de la grande migration du $\mathrm{xxI}^{\mathrm{e}}$ siècle ${ }^{4}$ », le mouvement de récupération des enfants eurasiens est terminé. Mais comment ne pas lier ces deux moments de l'arrivée en France de populations du Sud-Est asiatique ballottées par des politiques menées par 
des régimes pourtant bien différents, exerçant l'un et l'autre un biopouvoir touchant à la vie de milliers d'hommes, de femmes et d'enfants?

Présentés comme des «rapatriements » d'enfants de sang français, le déplacement des métis est lié à la fin de la présence française en Indochine, à la décolonisation, donc à tout un ensemble complexe de questions sur les plans politiques et diplomatiques, économiques et sociaux, démographiques et humains. Cette contribution vise à évoquer les pratiques mises en œuvre pour acculturer les enfants, les débats qu'elles ont suscités dans les années 1960, les mémoires qu'elles ont produites depuis les années 1980.

3 Pour cerner une réalité complexe, les sources mobilisées sont de natures variées. Les archives publiques et les documents de la FOEFI permettent d'appréhender la position des acteurs institutionnels. Un registre inédit d'identification des enfants eurasiens nés autour de la base de Seno (Laos) permet une étude de cas spécifique. Les témoignages de deux responsables de l'Association pour la protection des enfants au Laos (APPEL), permettent de voir une autre manière de prendre en charge les enfants. Les paroles des anciens pupilles de la FOEFI publiées sur le site Internet de leur association et dans leur publication Grain de riz sont précieuses.

4 À partir de ce corpus, il est possible de définir et de comprendre le contexte géopolitique, diplomatique et social dans lequel s'inscrit le sort des enfants eurasiens et en quoi leur déplacement relève d'une réflexion et de pratiques biopolitiques. Les différents modes de placement des enfants en France et les moyens d'acculturation mis en œuvre font l'objet de débats. Les histoires personnelles et les mémoires que conservent les « anciens » une fois devenus adultes montrent la complexité d'un épisode traumatique.

\section{Réflexions biopolitiques autour des enfants eurasiens}

\section{La question des métis en Indochine}

Dès l'implantation de la présence française en Indochine au xixe siècle, des Français, colons, fonctionnaires et surtout soldats, eurent des relations avec des femmes du pays. De ces unions mixtes amoureuses ou forcées, passagères ou plus durables, naquirent les premiers métis franco-indochinois. Pourtant, pendant longtemps les études sur la société indochinoise les ont ignorés 5 . Si le père français avait épousé une indochinoise et reconnu l'enfant, ce dernier prenait la nationalité du père. Mais si le père français était déjà marié et donc ne pouvait pas reconnaître l'enfant, ou s'il s'en désintéressait, celui-ci prenait la nationalité de la mère. Les hommes qui s'attachaient à une femme et à ses enfants ne pouvaient les emmener avec eux lors de leurs mutations. De nombreuses mères indigènes restaient donc seules avec leurs enfants et avaient bien des difficultés à assurer les soins nécessaires, sans parler de la pression sociale pesant sur elles. Le plus souvent elles les abandonnaient auprès des œuvres tenues par des congrégations religieuses françaises : les sœurs de Saint-Paul de Chartres et les sœurs de la Providence de Portieux arrivées dans les années 1860 et 1870, les religieuses de Notre-Dame-des-Missions et les sœurs de Saint-Vincent-de-Paul installées plus tardivement dans les années $1920^{6}$.

6 Par ailleurs, des Français d'Indochine créent des sociétés laïques locales pour venir en aide aux enfants eurasiens et assurer leur promotion : la Société d'assistance aux enfants franco-indochinois créée en 1897 au Tonkin, la Société de protection des métis en 1905 (Annam), la Société de protection de l'enfance de Cholon en 1908 (Cochinchine), la Société 
d'assistance aux métis en 1908 au Laos et la Fondation Gravelle, du nom de son bienfaiteur, en 1909 (Cambodge). De leur côté, les autorités françaises en Indochine étaient conscientes du problème de prestige que la question des enfants métis posait à la métropole mais également du potentiel démographique qu'ils représentaient.

Un décret du 8 novembre 1928 règle la situation des enfants non reconnus par un père mort au combat, décédé ou ayant abandonné sa compagne et son ou ses enfants. Il fixe en effet le statut des métis en disposant notamment que « tout individu, né sur le territoire de l'Indochine de parents dont l'un, demeuré légalement inconnu, est présumé de race française, pourra obtenir, conformément aux dispositions du présent décret, la reconnaissance de la qualité de Français » (article 1). Emmanuelle Saada a montré les implications de ce décret après en avoir décrypté toutes les dimensions et tous les termes, dont celui problématique de "race française ». Pour être considéré de "race française», l'enfant métis devait avoir du sang français dans les veines, mais aussi être socialisé dans un milieu de "culture française ${ }^{7}$ ». La mise en application de ces dispositions supposait bien sûr de recenser ces enfants, de les placer et les éduquer dans les institutions religieuses avec l'accord de leur mère. Ces établissements se chargeaient des démarches nécessaires afin qu'ils puissent obtenir la nationalité française.

Le gouverneur général de l'Indochine, Jules Brévié (1937-1939), portant attention aux métis, donna son nom à une fondation alimentée par des prélèvements sur le pari mutuel, sur les droits de l'alcool européen et sur plusieurs produits d'importation. La Fondation Jules-Brévié, créée en août 1939, devint la principale source de financement de la prise en charge des enfants eurasiens par les œuvres ${ }^{8}$. Quelques semaines plus tôt, une autre institution avait été créée par Brévié à Dalat (Annam) : l'École des enfants de troupe eurasiens, école préparatoire militaire qui permit de soulager la charge des autres institutions puisqu'elle était destinée à accueillir: "les enfants nés sur le territoire de l'Indochine, de père demeuré légalement inconnu mais présumé de race française ; les enfants nés sur le territoire d'Indochine d'une mère indigène et d'un père de race française ${ }^{9}$ ». Des "petits gradés» sortis de l'école serviront pendant la guerre d'Indochine, puis ensuite en Algérie.

\section{« Rapatrier » les enfants eurasiens après la fin de l'Indochine française}

9 À la fin de la seconde guerre mondiale et après le départ des Japonais d'Indochine, les pensionnats furent restaurés par le général Leclerc qui imposa un changement de nom à la Fondation. En effet, d'avril 1942 à mars 1943, Brévié avait été ministre de l'Outre-mer et des Colonies dans le gouvernement de Pierre Laval et il fut déchu de son grade de gouverneur général honoraire des Colonies en janvier $1945^{10}$. Ainsi naquit la Fondation eurasienne présidée par William Bazé, planteur d'hévéas, lui-même eurasien né en 1899 en Annam, engagé dans la résistance en $1943^{11}$, ce qui ne l'empêchait pas de rendre hommage à l'action sociale de Brévié avec qui il avait commencé à s'intéresser au sort des métis. Ne pouvant avoir d'enfants, les Bazé ont recueilli et élevé jusqu'à leur majorité 14 petits eurasiens orphelins ${ }^{12}$. La nouvelle organisation prit le nom de Fondation fédérale eurasienne puis de Fédération des œuvres de l'enfance française d'Indochine (FOEFI) dont les statuts furent approuvés par décret le 25 juin 1953 et qui fut reconnue d'utilité publique $^{13}$. 
10 Pendant la guerre d'Indochine, la présence d'un important corps expéditionnaire accrut considérablement le nombre des naissances d'enfants métis en même temps que s'aggravait la question de leur place dans la société indochinoise puisqu'ils étaient des enfants de soldats coloniaux venus reconquérir le pays. Les actions des associations s'accentuèrent donc. Les chiffres avancés par Bazé en 1952 font état de 300000 enfants métis - eurasiens et africasiens - en Indochine, ce qui paraît très exagéré mais significatif du sentiment d'explosion du phénomène ${ }^{14}$. À partir de 1947, la fédération commença à évacuer des enfants eurasiens vers la France où Marguerite Graffeuil, veuve du Résident supérieur en Annam de 1934 à 1940 (Maurice Graffeuil), organisa à son retour en métropole leur accueil avec l'aide du ministère de la France d'Outre-mer. Grâce à ses connaissances et à ses relations avec des personnalités politiques et des institutions religieuses, elle obtint des subventions qui permirent à la fédération d'acheter des propriétés où furent accueillis les enfants ${ }^{15}$.

11 Après la chute de Dien Bien Phu et les accords de Genève (1954) qui consacrèrent la division en deux États indépendants du Viêtnam, William Bazé et Marguerite Graffeuil continuèrent à s'occuper des enfants eurasiens rapatriés. Il s'agissait d'un enjeu considérable de la décolonisation. Un accord entre les gouvernements français et vietnamien permit en effet aux métis eurasiens et africasiens recueillis par une œuvre française de s'établir en métropole «en raison de l'éducation qu'ils ont commencé à recevoir ${ }^{16}$ ». Le déplacement de ces enfants est de la responsabilité de la FOEFI, jamais directement de celle de l'État, hormis pour ceux de l'École d'enfants de troupe eurasiens qui quitta Dalat en 1954 et se réinstalla en France en 1956 (à Fréjus puis à Autun) avant de fermer définitivement ses portes en 1960 après le départ du dernier enfant de troupe eurasien ${ }^{17}$. La FOEFI, organisation non étatique, se trouve donc investie d'un biopouvoir délégué par l'État qu'elle exerce avec toute autorité.

12 Les responsables de la FOEFI s'installent en 1954 dans un appartement au 7 de la rue Washington à Paris. Pour eux, il s'agit de sauver des milliers d'enfants, sans avenir dans leur pays de naissance puisqu'ils seraient rejetés sans doute par leur famille, sûrement par l'ensemble de la population, et de leur donner en France un enseignement et une formation professionnelle. Le déchirement des mères qui confient ainsi leurs enfants est considéré comme un acte d'amour les sauvant du mauvais sort qui les attend dans les deux Viêtnam... surtout celui du Nord ${ }^{18}$. À partir de 1954, ces mères doivent signer un «certificat de décharge » à la FOEFI indiquant que l'organisation a le droit « sans accord ultérieur de ma part, d'envoyer mon enfant en France ou dans n'importe quel pays de l'Union française, pour y poursuivre ses études ou acquérir une formation professionnelle ${ }^{19}$ ». L'accord de la mère ne concernant que l'éducation de son ou ses enfant(s), l'adoption de ces enfants eurasiens "rapatriés " par la FOEFI est interdite. Toutes les mères qui refusent de confier leurs enfants les gardent auprès d'elles.

\section{Le cas des enfants de la base de Seno}

13 Au début de l'année 1962, le consul de France à Vientiane invite les responsables français d'une association qui secourt des enfants vietnamiens, laotiens et chinois à porter attention aux enfants métis dont personne ne s'occupe. Selon le fonctionnaire, ils seraient plus de 500 au Laos, surtout autour des bases militaires françaises. Jusque-là «le problème des enfants eurasiens nous avait complètement échappé » avoue René Péchard (1912-1988), un Français installé depuis de nombreuses années au Laos et qui préside 
l'Association pour la protection de l'enfance au Laos (APPEL) ${ }^{20}$. C'est ainsi qu'il commence à s'intéresser au sort des enfants de pères soldats français, notamment ceux qui vivent autour de la base militaire de Seno (près de Savannakhet), maintenue française d'après les accords de Genève. Le colonel Dunoyer de Segonzac, commandant de la base, accepte de mettre tout en œuvre pour ces enfants ${ }^{21}$. Prévue pour deux divisions, avec un terrain d'aviation, une importante infirmerie, etc., la base «hébergeait et protégeait 3500 Vietnamiens (hommes, femmes et enfants) qui avaient suivi le corps expéditionnaire jusqu'au bout ", écrit a posteriori Jacques Suant qui commanda la base en 1964-1965 ${ }^{22}$. Évidemment, la base ne pouvait plus jouer de rôle militaire - toutes les armes avaient été évacuées -, mais «il y avait lieu de l'utiliser au service du rayonnement de la France auprès des populations qui croyaient encore en elle ». Aussi, l'infirmerie est transformée en hôpital pour tous, y compris les populations civiles, et une école ouvre pour les enfants de la base et au-delà (jusqu'à 1000 élèves), assurée par des sous-officiers et des épouses d'officiers ${ }^{23}$.

Une autre action visa à prendre en compte la situation « des orphelins (de père surtout) qu'on rencontrait tous les jours ». Il s'agissait en premier lieu de leur donner l'état civil qui leur faisait défaut, prélude absolument nécessaire pour que ces enfants métis soient considérés comme français et qu'ils puissent être pris en charge en France par la FOEFI. Pour ce faire, les commandants de la base firent recenser les enfants afin : «a) de les soigner, b) de les christianiser, c) de leur donner un état civil, et dans la mesure du possible, de leur assurer un avenir, donc de les envoyer en France (avec l'accord de la mère dans tous les cas ${ }^{24} »$.

La lecture du registre constitué est très instructive sur la manière de procéder. Pour chacun des 267 enfants recensés une fiche est établie avec sa photo indiquant « nom et prénom, date et lieu de naissance officiels », "date et lieu de naissance réels ", " nom d'origine ", «nom du père réel et situation ethnique ", "nom et race de la mère ", "moyen de naturalisation française ». Le but de l'entreprise est de faire de ces enfants des Français. Parmi les plus âgés, nés dans les années 1945 et 1946, un certain nombre s'engage dans l'armée française. L'APPEL se charge de faire le lien entre le service social de l'armée, le consulat de France et la FOEFI.

Pour la période allant de 1963 à 1965, le registre mentionne 16 adoptions. En effet, indique Jacques Suant, "j'ai accentué le mouvement, et l'ai étendu jusqu'à des "adoptions", le bouche à oreille fonctionnant. Je n'ai pas toujours eu l'appui des services consulaires de l'ambassade et j'ai bien conscience d'avoir enfreint la loi française de l'époque ». En effet, des enfants abandonnés par leurs mères ont été reconnus par des militaires français - qui n'en étaient pas les pères - puis les ont abandonnés officiellement afin qu'ils puissent être adoptés. Mais le commandant poursuit : "à vrai dire, je ne regrette rien » et il se rappelle « les briscards de la coloniale à qui j'avais fait donner des cours de puériculture (langes, biberons, etc.) à l'infirmerie et qui ont ramené dans leurs bras en France des dizaines de bébés et d'enfants ${ }^{25}$ ». Grâce à la Mission militaire française en Indochine et au colonel Dunoyer de Segonzac, sur les 267 enfants recensés, 194 enfants partent pour la France en 1963 où ils sont pris en charge jusqu'à leur majorité par la FOEFI. Les enfants dont les mères s'opposent à leur départ restent au Laos $^{26}$. Certains d'entre eux partiront plus tard. Sur le registre de Seno, quelques fiches mentionnent des précisions sur l'adoption de l'enfant. Ainsi, cette petite fille née en novembre 1962 d'une mère vietnamienne et d'un père sergent français rapatrié en France. Elle est adoptée en 1963 par une famille française installée à Vientiane. Sa demi- 
sœur, née en 1957 de la même mère et d'un autre militaire français, déclarée sous un autre nom que sa sœur, est adoptée en 1963 par une famille de métropole ${ }^{27}$.

Dans cet épisode et dans le contexte postcolonial français en Indochine, biopolitique, populationnisme et bien d'autres considérations se mêlent. L'envoi des enfants eurasiens en France, qui peut être considéré comme une fin pour les acteurs qui organisent leur départ, n'est pour les principaux intéressés qu'un commencement comme pour la FOEFI qui se charge de les accueillir en France.

\section{De la meilleure méthode d'acculturer les enfants eurasiens}

\section{Les foyers de la FOEFI}

Un des buts de la FOEFI est de poursuivre l'histoire coloniale de la France. Les jeunes générations qu'elle forme sont censées donner à l'État des éléments de valeur qui maintiendront avec les pays d'Outre-mer les liens noués au cours d'une présence séculaire. Vivant et grandissant ensemble, ils n'oublieront pas leur double identité : « Les enfants d'origine mixte élevés dans l'amour de leur patrie et de leur pays natal sont un trait d'union indissoluble entre deux races et deux civilisations" disait William Bazé. Pour cela, ils doivent demeurer ensemble, entre eux.

19 En 1955, le commandant Grolleau, délégué général de la FOEFI, acheta pour la fédération plusieurs biens immobiliers: les châteaux du Pont de Cisse à Vouvray, La Source à Semblançay (1955), Rilly à Beaumont-sur-Loire, l'hôtel particulier Magenta à Tours. Grâce à ses contacts dans l'armée, il récupéra 200 lits et 400 couvertures installés dans l'urgence pour permettre au foyer de Vouvray d'accueillir le premier convoi d'enfants qui arrivait de Saigon par avion... Beaucoup d'autres enfants suivront par bateau. Les Eurasiennes, qui ont été beaucoup moins nombreuses à être envoyées en France - car n'ayant pas la même importance au regard des acteurs de ce mouvement ? - sont accueillies à partir de 1949 à Saint-Rambert-en-Bugey (Ain) où Marguerite Graffeuil - « marraine » - a sa chambre. Des adolescentes sont placées dans d'autres institutions à Monaco, à Dreux, etc. Les directeurs des foyers de Vouvray et de Semblançay sont des anciens de l'Indochine qui connaissent bien la réalité des enfants métis ${ }^{28}$.

Dès l'arrivée, tout est fait pour montrer aux métis qu'ils doivent tourner une page de leur vie. Certains de leurs objets personnels qui les rattachent à leurs origines sont confisqués, il leur est interdit de parler vietnamien sous risque de punition, des fratries sont séparées. Des enfants fuguent pour retourner à Marseille... où ils ont débarqué, avec cette interrogation : «Je suis né au Viêtnam, qu'est-ce que je fais en France ${ }^{29}$ ?"

21 À Vouvray et à Semblançay, des écoles primaires ont été ouvertes à l'intérieur des foyers, certains enfants demeurent entre eux en permanence, d'autres fréquentent les écoles des communes avoisinantes. Traités de " chinetoques ", voire de "bâtards », ils ont honte de ne pas avoir de parents. Une habitante de Semblançay, dont les parents travaillaient au foyer, témoigne qu'au début les habitants du village craignaient ces "viets» qui débarquaient en nombre et allaient chaparder dans les jardins, « mais petit à petit ils se sont "civilisés" [...] ils ont été à l'école avec nous les enfants du village, ils ont fait leur communion avec nous, et je pense que par la suite tout le monde les a acceptés et ensuite bien appréciés même ! Ils faisaient partie des habitants de Semblançay ${ }^{30}$ ! » 

cours de l'été 1957, le député de la circonscription et le préfet enjoignent la FOEFI de prendre des mesures contre les larcins et déprédations commis par les pensionnaires. Le 15 septembre les habitants manifestent leur hostilité devant le foyer. Pour les responsables de la fédération, «quelques faits regrettables ne devaient, en aucun cas, porter atteinte à une œuvre qui compte à son actif tant de brillants résultats ». La dispersion des 97 enfants est donc organisée dans 27 institutions différentes à Paris, de Saint-Malo à Brive-la-Gaillarde, d'Orléans à Marseille ${ }^{31}$.

L'année 1957 est marquée par un autre épisode au foyer de Semblançay qui accueille alors une soixantaine d'enfants. L'été est lourd et la situation tendue. Les plus grands revendiquent sur l'argent de poche, les sorties, la nourriture, contre la sieste obligatoire... Ils parlent vietnamien entre eux, s'échauffent. Le 2 août, deux gendarmes sont appelés par le directeur pour calmer les esprits, ils sont accueillis à coups de pierres, il y a bousculade, l'un d'eux sort son arme et tire en l'air. C'est la panique générale; certains enfants fuguent, bien vite repris par des forces de l'ordre arrivées en nombre ${ }^{32}$. La FOEFI considéra cet épisode comme un «incident grave » et son président "sermonna les enfants avec sévérité en s'exprimant d'abord en français, puis en vietnamien ». Les neuf grands considérés comme les meneurs furent immédiatement évacués du foyer ${ }^{33}$. L'un d'eux fut confié aux Apprentis-Orphelins d'Auteuil, tandis que son frère, plus jeune, fut transféré au foyer de Vouvray ${ }^{34}$.

\section{D'autres méthodes et moyens mis en œuvre}

La FOEFI ne manque pas d'argent. Les enfants partent en colonies de vacances à la mer, à l'étranger (Espagne, Yougoslavie, etc.), font du ski, sont bien habillés. Ils sont élevés et éduqués pour s'insérer dans la société française et devenir des Français comme tous les autres, avec de bonnes situations. La fédération se donne pour mission de faire grandir ensemble les enfants eurasiens «rapatriés" afin d'atténuer le choc brutal de leur changement de vie. Le but de ce choix éducatif est une acculturation collective, générationnelle même. Mais les problèmes d'acceptation par le voisinage ou les tensions internes aux foyers de la FOEFI poussent celle-ci à envisager d'autres moyens de prise en charge pour les métis. La dispersion dans une multitude de pensionnats de quelques enfants à la fois semble une voie médiane entre une trop grande concentration des enfants et un isolement non encadré qui est considéré comme la pire des options. Hormis les foyers, la plupart des enfants sont accueillis en petits groupes dans quelques centaines de petites structures. Des filles ont été placées dans des familles qui les ont élevées comme leurs propres enfants. Certains pères suivent discrètement l'évolution de leurs enfants. Des mères envoient des courriers, certaines, arrivées plus tard en France, rendent visite à leurs enfants dans les foyers.

Les Eurasiens et les Africasiens constituent un objet d'étude pour les anthropologues. En 1967 sont publiés les résultats d'un travail de recherche visant à mieux définir «des hybrides de première génération (F1), des "demi-sang" comme on disait autrefois; il s'agit de sujets dont la mère est vietnamienne et le père citoyen français ». Les "sujets » sont examinés dans les foyers de Vouvray, Semblançay et Saint-Rambert, mais il est précisé que "dans quelques cas, les enfants ont été examinés dans leur famille, en particulier les Afrasiens [sic] ramenés au Sénégal par leurs pères. Car l'étude ne porte pas seulement sur des métis entre Blancs et Jaunes, mais aussi sur des métis entre Noirs et 
Jaunes $^{35}$ ». Les enfants et adolescent-e-s ressentent une profonde humiliation à être "mesurés comme du bétail ${ }^{36}$ ".

Pour l'APPEL, le rassemblement des enfants eurasiens dans les foyers de la FOEFI ne semble pas le meilleur moyen de les intégrer dans la nation française. Bien qu'il exprime en 1963 une réelle gratitude à l'égard de la fédération pour la prise en charge de certains enfants de Seno, lors d'un entretien accordé en 1989, Péchard se montre très critique :

«Les jeunes Eurasiens des années 1960 ont un foyer et un travail, certes, mais ils gardent au cœur un regret, celui de n'avoir pas ou peu rencontré de familles qui les auraient accueillis comme leurs propres enfants quand, après les péripéties et les incertitudes qu'on sait, les autorités françaises les ont rapatriés dans l'hexagone ${ }^{37}$. »

Cette prise de position entraîne des réactions à la FOEFI. Un magistrat qui a été en poste au Laos dans une brève « histoire de la FOEFI au Laos » évoque à propos de Seno « deux organismes, non qualifiés et sans expérience pour traiter les problèmes » des enfants eurasiens. Et de citer le service social de l'armée et l'APPEL, et de dénoncer le placement d'enfants «sans la présence à leurs côtés de camarades de même origine, auprès desquels ils pouvaient trouver appui et réconfort ${ }^{38} »$.

Estimant que seul l'accueil dans des familles permet la véritable intégration des enfants en France, l'APPEL développe des formes de prise en charge différentes de celles de la FOEFI. En 1964, les personnes intéressées par l'action de l'APPEL envers les enfants laotiens reçoivent un imprimé intitulé «Parrainage et Adoption ». Jean-Claude Didelot, qui à partir de 1968 anime les activités de la branche française de l'association (ASPELFrance), les définit en fonction des enfants à aider, de leurs origines, de leurs âges et de leurs besoins. Le principe essentiel est que tout enfant se retrouve au sein d'une famille métropolitaine ${ }^{39}$. Un hommage rendu à René Péchard par le chef de la section consulaire française à Vientiane indique qu'il a "sauvé de la misère plus de trois cents jeunes Français en les envoyant en France pour les placer, soit dans des œuvres françaises, soit dans des familles d'accueil ${ }^{40} »$. Un rapport parlementaire de 1974 indique que «l'ASPEL aurait assuré des placements familiaux en France à plusieurs centaines d'enfants du Laos ${ }^{41} »$.

\section{Les mémoires vives d'une génération transplantée}

\section{La fin d'une époque en Asie du Sud-Est et en France}

En août 1972, la FOEFI reçoit de l'État une subvention de 4300000 francs qui lui permet de régler toutes ses dettes. Mais en raison de la diminution du nombre des Eurasiens relevant de la fédération, l'État demande la cessation de ses activités avant le 31 décembre 1976. Les derniers "rapatriements » de mineurs eurasiens ont donc lieu cette année-là ${ }^{42}$. Ce sont surtout des adolescents qui deviennent vite majeurs, en même temps que les enfants arrivés au lendemain de la guerre d'Indochine. La mission de la FOEFI s'achève après avoir pris en charge environ 4500 enfants. De 1977 à 1983, sans aucune subvention, la FOEFI continua à aider ses anciens pupilles. Ceux qui à leur majorité ont laissé leurs coordonnées ou les ont actualisées reçoivent leur dossier personnel établi par la FOEFI. Les autres dossiers sont confiés au Centre des archives de l'Outre-mer (CAOM) à Aix-en-Provence. C'est là-bas que les anciens de la FOEFI peuvent retrouver, parfois, quelques traces de leurs ascendants, de leur vie ${ }^{43}$, tout comme les enfants de la ZFO en Allemagne peuvent faire le même retour en arrière dans des archives 
de l'occupation française en Allemagne conservées désormais à la Courneuve (ministère des Affaires étrangères).

Les années 1970 sont aussi celles de la fin de la guerre du Viêtnam. 1975, avec la chute de Saigon, le retrait américain et la question des Amérasiens renvoie à 1954 avec Dien Bien Phu, le départ des Français et les Eurasiens. La logique biopolitique est également à l'œuvre du côté américain. Le 3 avril, le président américain Gerald Ford déclenche Operation Babylift dont l'objectif est d'évacuer les enfants nés de pères GI, ceux qui sont en cours d'adoption par des Américains et plus généralement les orphelins de la guerre dont le nombre est estimé à 70000 . Aussitôt, des œuvres françaises qui travaillent au Viêtnam demandent à pouvoir faire partir « leurs » enfants vers les États-Unis ${ }^{44}$. Dès le 4 avril, 52 enfants de l'hospice des sœurs de Saint-Paul de Chartres sont embarqués grâce à Friends for All Children dans un avion Galaxy C-5 de l'US Air Force. Juste après avoir décollé, l'appareil connaît une avarie qui le contraint à faire demi-tour. Il s'écrase non loin de l'aéroport de Saigon. On dénombre plus de 150 morts dont près de cent enfants sur les 310 à 330 personnes embarquées ${ }^{45}$.

31 Malgré la précipitation et le désordre, l'ambassadeur de France prend le temps de pointer les objections qui s'expriment fortement face au Babylift, que la radio de Hanoi qualifie de «trafic d'enfants». L'accident du Galaxy renforce le sentiment de malaise des autorités de Saigon qui ne peuvent que consentir au Babylift face aux pressions américaines. "Ce douloureux problème a une dimension politique que je me dois dans les présentes circonstances de relever », conclut le diplomate ${ }^{46}$. Pour Le Monde, « rarement sans doute, ne fut aussi crûment mise à nue l'ambiguïté des accès de générosité collective »; « le sort de centaines d'enfants s'est trouvé fixé en quelques heures par des bonnes volontés parfois plus soucieuses des mauvaises consciences occidentales que du sort des enfants ${ }^{47}$ ». La situation est tout autre aux États-Unis où le Babylift a été couvert par tous les médias américains comme un fait de guerre compensant les images désastreuses de la chute de Saigon. L'opération est considérée comme une bataille gagnée dans la guerre perdue du Viêtnam, peut-être un des rares épisodes du conflit et de l'engagement américain à valoriser ${ }^{48}$.

La fin de la guerre du Viêtnam et la victoire des communistes poussent certaines mères qui, bien des années plus tôt, avaient confié leurs enfants à la FOEFI à reprendre contact et à leur demander qu'ils les fassent venir en France au motif qu'elles risquent leur vie dans le Viêtnam communiste ${ }^{49}$.

\section{L'association FOEFI}

Au printemps 1987, pour fêter ses 40 ans, un ancien de la FOEFI a l'idée de réunir un groupe d'amis eurasiens. Grâce au Minitel, il réussit à contacter beaucoup d'anciens de la "promo 1947 ». Dans la foulée de cette rencontre, où étaient également présents le directeur du foyer de Semblançay, un éducateur et une assistante sociale, en juillet ont lieu les premières grandes retrouvailles dans l'enceinte du château de la Source. Une association loi 1901 est ensuite créée. Une identité FOEFI se dessine entre anciens : des souvenirs de l'Indochine ou du Viêtnam, le foyer et/ou les pensions, la solidarité et la camaraderie comme moyens de se soutenir, d'avancer dans la vie. L'un d'eux témoigne : «mes enfants ont trouvé que j'avais "changé" depuis ces retrouvailles, il devait me manquer la chaleur de mes copains, en fait ils ont été ma seule famille durant mes 10 années de FOEFI $^{50} »$. 

activités de l'association. La plume est donnée autant aux éducateurs qu'aux anciens pupilles de la fédération. Des hommages sont rendus aux "parrains » et " marraines » (les responsables des foyers) sans pour cela éviter le débat sur la sévérité des encadrements ${ }^{51}$. "L'époque plus ou moins heureuse de la vie dans les foyers ou les pensionnats » est au centre de la publication avec de nombreuses photos, mais aussi les fêtes vietnamiennes (Têt), la cuisine, la littérature, etc. La journée festive annuelle rassemblant les membres de l'association et leurs familles est l'activité principale de l'association.

La préservation et la transmission de la mémoire passe par des gestes symboliques comme la pose d'une plaque commémorative à Semblançay sur laquelle est écrit: «De 1955 à 1967 à la Source, des garçons de la FOEFI ont vécu leur enfance en gagnant l'affection de tous ${ }^{52}$.» Les Eurasiennes se rassemblent pour la première fois à SaintRambert-en-Bugey en 2010 ${ }^{53}$. Il existe une amicale des AET-Dalat (Anciens Enfants de Troupe) qui se réunit tous les ans à Vogüe en Ardèche ${ }^{54}$. camaraderie et l'expression du sentiment d'avoir été sauvés, mais le film est surtout un tournant important dans la mémoire des anciens de la FOEFI et leur association ${ }^{55}$. Ils expliquent :

« Nous avons très peu parlé de notre blessure commune : de l'absence de nos mères et de nos pères. Cette blessure était si profondément enfouie en nous que très peu d'entre-nous en ont parlé, à leur femme, et surtout à leurs enfants. C'est pour rompre ce silence que nous avons accepté de témoigner [...]. À travers le film, nous avons voulu dire que nous avons tous eu à porter cette blessure et qu'il est temps aujourd'hui d'en parler, entre-nous, avec nos enfants qui eux aussi ont souffert de nos silences et qui ont besoin de savoir ${ }^{56}$.»

Pour la génération des "quarterons »- comme ils s'appellent eux-mêmes -, le film est une véritable révélation sur ce qu'ont vécu leurs parents et sur une histoire et des origines qui sont aussi les leurs ${ }^{57}$.

\section{Des plaies encore ouvertes}

38 Si les histoires individuelles et personnelles sont aussi nombreuses que les enfants eurasiens eux-mêmes, les cicatrices sont les mêmes, plus ou moins refermées. Le départ du pays natal a été douloureux, surtout pour ceux qui attendaient en vain que leurs mères viennent leur dire au revoir à l'embarquement. Pour beaucoup c'est un voyage comme un autre n'ayant pas conscience de la distance séparant l'Indochine de la France. Sur le bateau, ils côtoient des soldats français rentrant en métropole, chacun se choisit un père fictif. Plusieurs décennies après ils pensent que leurs mères n'avaient pas le choix et qu'elles savaient qu'elles ne reverraient pas leurs enfants en leur disant adieu. L'hommage aux mères devient plus présent dans Grain de riz, la quête du père, de frères et sœurs, devient plus fréquente, du moins est davantage exprimée ${ }^{58}$.

Si le déracinement est évoqué dans le film de Philippe Rostan, l'arrachement est le thème principal du documentaire Né sous $Z$ de Frédérique Pollet-Rouyer (2010) qui suit la quête de Robert pour connaitre ses origines et comprendre les motivations de la politique dont les enfants eurasiens ont fait l'objet. Découvrant les réalités du mouvement de « rapatriements », justifié par la nécessité de sauver ces enfants, il s'interroge : « est-ce 
qu'on avait besoin d'être sauvés $?^{59} »$ Ces documentaires provoquent un véritable débat au sein des foefiens et foefiennes, comme ils s'appellent entre eux. Les responsables de foyers, éducateurs et assistantes sociales sont critiques sur une manière de regarder la réalité des années 1950 et 1960 avec les yeux, les mentalités, les valeurs d'aujourd'hui. Pour les anciens pupilles, une parole s'est libérée, ils veulent pouvoir exprimer leurs déchirures en même temps que leur gratitude ${ }^{60}$. Comme pour beaucoup d'autres épisodes traumatiques, le temps est nécessaire à l'expression du mal-être, à la résilience définie par Boris Cyrulnik comme "la capacité à se développer quand même, dans des environnements qui auraient dû être délabrants ${ }^{61} »$. Dans un livre paru très récemment intitulé L'Enfant de Seno, Laby Camara raconte son histoire. Africasien abandonné par son père tirailleur sénégalais, puis délaissé par sa mère vietnamienne, il grandit au Laos et à treize ans est envoyé en France. Il connaît la vie dans la rue, si loin de chez lui. Ces mémoires racontent aussi ses retrouvailles avec sa famille paternelle guinéenne, sa reconstruction malgré une vie impossible et montrent le pouvoir libérateur de la parole et de l'écriture ${ }^{62}$.

40 Si le Minitel a permis les premières retrouvailles dans les années 1980, l'Internet permet d'aller beaucoup plus loin dans les recherches et ce des deux côtés. Comme le mouvement québécois Retrouvailles, initié par des mères voulant retrouver la trace d'enfants qu'elles ont dû abandonner des décennies plus tôt ${ }^{63}$, des mères vietnamiennes recherchent leurs enfants qui ont grandi et fait leur vie en France. La chaîne de télévision vietnamienne VTV4 réalise une fois par mois une émission intitulée "Comme s'il n'y avait pas eu de séparation " au cours de laquelle des mères et des enfants se retrouvent, des frères et sœurs aussi. Ainsi, Jean, René et Jacqueline Weber retrouvent - 56 ans après - leur sœur aînée Huong perdue de vue depuis $1954^{64}$.

\section{Conclusion}

41 Les « rapatriements » en France d'enfants eurasiens, afin de leur assurer une éducation et un avenir, ont été laissés aux soins de la FOEFI. C'est l'une des caractéristiques de la politique de population liée à la décolonisation de l'Indochine : l'intention, la mise en pratique et le suivi de l'acculturation générationnelle visée ont été le fait d'un acteur privé auquel l'État a délégué un pouvoir qui a bouleversé la vie de milliers de personnes sur plusieurs générations: mères (et dans une moindre mesure pères), enfants et descendants.

L'aller simple pour l'intégration organisé par la FOEFI renvoie à de nombreuses questions que pose la biopolitique notamment sur l'instrumentalisation des enfants dans une entreprise post-coloniale. Les acteurs eux-mêmes, organisateurs de cette migration contrainte, se sont interrogés sur les meilleures méthodes pour parvenir à l'acculturation des enfants eurasiens. Ces derniers sont devenus, sans qu'on leur demande quoi que ce soit, les objets d'un programme raisonné visant à faire d'eux une courroie de transmission avec l'ancienne colonie. L'inéluctabilité de la décolonisation en Afrique après l'Indochine et la guerre du Viêtnam perdue par les Américains ont rendu illusoire cet objectif devenu impossible à atteindre. Si l'échec de l'entreprise biopolitique postcoloniale est ainsi patent, il contraste avec une certaine réussite de l'éducation donnée à ces enfants et à ces jeunes. Ceux-ci ont grandi, plus ou moins ensemble, dans des institutions spécialement conçues pour eux ou accueillant aussi d'autres enfants. Bien 
qu'il soit impossible d'avancer des chiffres précis, la plupart d'entre eux sont devenus des adultes « comme les autres ", ainsi qu'ils aiment à se définir eux-mêmes.

Après la séparation liée à la vie active et aux itinéraires personnels des uns et des autres, la maturité a poussé certains à retrouver leur enfance, leurs amis. L'association FOEFI a ainsi été créée et a grandi avec la camaraderie pour ciment et une identité commune comme parcours de vie. Encore le temps d'une génération, celle des petits-enfants et de leur intérêt pour le passé de leurs ascendants, et sont remontés d'autres souvenirs mis en lumière par des livres, des films. D'autres quêtes que celle de l'amitié retrouvée sont poursuivies aujourd'hui pour retrouver des frères et sœurs, si ce n'est une mère ou un père dont beaucoup sont morts.

Pour la génération des enfants de la FOEFI, dont les plus anciens ont dépassé soixante-dix ans, le recul est déterminant pour porter sur leur enfance et le traitement dont ils ont fait l'objet un regard apaisé : «Si la lucidité acquise nous pousse à reconnaitre la chance que nous avons eue d'être restés en vie grâce à la FOEFI, elle nous permet aussi de dire, sans ingratitude, que tout n'a pas été parfait dans le meilleur des mondes possibles ${ }^{65}$. » SHAPE

\section{NOTES}

1. Inconnu présumé français réalisé par Philippe ROSTAN, France, $2009,90 \mathrm{mn}$, diffusé sur France Ô en avril 2010 ; Né sous $Z$ réalisé par Frédérique POLLET-ROUYER, France-Belgique, 2010, 75 mn, diffusé sur France 2 en novembre 2011.

2. SAADA Emmanuelle, Les enfants de la colonie. Les métis de l'Empire français entre sujétion et citoyenneté, Paris, La Découverte, 2007, 335 p. Publication issue d'une thèse soutenue en 2001 à l'EHESS; d'autres universitaires avaient également proposé des études ou des évocations sur les métis, notamment: Rolland Dominique, De sang mêlé. Chronique du métissage en Indochine, Toulouse, Elytis, 2006, 382 p.

3. DÉNĖCHERE Yves, "Des adoptions d'État: les enfants de l'occupation française en Allemagne, 1945-1952 ", Revue d'Histoire Moderne et Contemporaine, n 57-2, avril-juin 2010, p.159-179. Paul-André ROSENTAL, L'intelligence démographique. Sciences et politiques des populations en France (1930-1960), Paris, Odile Jacob, 2003.

4. FoUCAULT Michel, «Le problème des réfugiés est un présage de la grande migration du XXI e siècle ", Dits et écrits tome III 1976-1979, Paris, Gallimard, 1994, p. 798-800. Cité par Anna SIMONE «Migrations» dans Lexique de biopolitique. Les pouvoirs sur la vie (collectif), Toulouse, Érès, 2009 (2006), p. 202-206.

5. MEYER Charles, La vie quotidienne des Français en Indochine 1860-1910, Paris, Hachette, 1985. Dans un chapitre intitulé "Pièges et sortilèges", l'auteur consacre plusieurs pages au "Charme des petites épouses ", sans jamais évoquer les métis nés de ces rencontres, p. 263-270.

6. DUFOURCQ Élisabeth, Les aventurières de Dieu. Trois siècles d'histoire missionnaire française, Paris, Jean-Claude Lattès, 1993, p. 406-408. 
7. SAADA Emmanuelle, Les enfants de la colonie..., p. 13, article 2 du décret du 8/11/1928.

8. Historique de la FOEFI présenté par William Bazé lors du Conseil d'administration de clôture de la FOEFI, le 7 novembre 1983. Consultable sur [

http://foefi.net/naissancedelafoefi.html

\section{]. L'histoire de la FOEFI et des œuvres qui l'ont précédée reste à écrire.}

9. Arrêté du gouverneur général de l'Indochine portant création de l'École des enfants de troupe eurasiens de Dalat, 27/06/1939, article 1.

10. Jules Brévié (1880-1964) a été définitivement privé de sa pension de retraite en mars 1945 et en mai il est déchu du droit de porter toute décoration française ou étrangère.

11. LACoutURE Jean, De Gaulle, 2 Le politique, Paris, Le Seuil, 1885, p. 157.

12. William Bazé a épousé Yvonne de Miribel, fille du Résident supérieur de France au Tonkin.

13. Sur tous les documents commémoratifs et pages Internet qui évoquent la FOEFI, sa création est datée de 1938, sans doute pour éviter de rappeler le nom de Jules Brévié.

14. SAADA Emmanuelle, Les enfants de la colonie..., p. 237.

15. Historique de la FOEFI présenté par William Bazé, op. cit., CA de la FOEFI, 7/11/1983.

16. SAADA Emmanuelle, Les enfants de la colonie..., p. 235-236.

17. L'histoire de l'école est mal connue, on en trouve une seule mention dans l'ouvrage collectif Historique des Enfants de troupe et des Écoles militaires préparatoires, Saint-Girons, Maury, 1963, $305 \mathrm{p}$.

18. Témoignage de Marguerite Graffeuil recueilli en avril 1987 par un de ses filleuls eurasiens. Consultable sur [http://www.foefi.net/].

19. SAADA Emmanuelle, Les enfants de la colonie..., p. 237.

20. Compte rendu de l'assemblée générale de l'APPEL, 28/11/1963. Ce document et beaucoup d'autres sont réunis sur le CD accompagnant le livre de Jean-Claude DIDELOT, Piété filiale. Des certitudes à la foi avec René Péchard, Paris, Éditions du Jubilé, 2004.

21. Jean-Claude DARRIGAUd et Jean-Claude DiDelot, Les enfants du Mékong, Paris, Fayard, 1989, p. 74.

22. Après sa retraite, Jacques Suant est devenu écrivain. Il a notamment publié : Rizières de sang (1970) et Viêtnam 1945-1970 (1972).

23. Archives privées, Registre des enfants eurasiens de Seno, déclaration préliminaire, p. 1 et 2 .

24. Ibid.

25. Ibid.

26. Compte rendu de l'assemblée générale de l'APPEL, 28/11/1963, op. cit.

27. Archives privées, Registre des enfants eurasiens de Seno.

28. «Lettre de $\mathrm{M}^{\text {me }}$ Teisserenc à ses enfants eurasiens (juillet 2010) ", Grain de riz, $\mathrm{n}^{\circ} 42$, p. 7 ; Souvenirs de Paul Susini, Grain de riz, n 44 , p. 12-13.

29. Témoignages dans le documentaire Inconnu présumé français.

30. Témoignage d'Anne-Marie Gauguin publié dans Grain de riz, n 40, mars 2010, p. 4-5. 
31. Archives de la FOEFI, compte rendu de la situation à Rilly (1957) dans Grain de riz $n^{\circ} 41$, juin 2010, p. 8 .

32. Témoignage d'un ancien de Semblançay, Grain de riz n 41, juin 2010, p. 4-5.

33. Archives de la FOEFI, compte rendu de l'incident de Semblançay (1957) dans Grain de riz, $\mathrm{n}^{\circ}$ 41, juin 2010, p. 7.

34. Témoignage d'un ancien de Semblançay, Grain de riz n 41 , juin 2010, p. 4-5.

35. Bulletins et Mémoires de la Société d'anthropologie de Paris, $\mathrm{XII}^{\mathrm{e}}$ série, tome 1, fascicule 1, 1967. Numéro thématique: Anthropologie des métis franco-vietnamiens, $114 \mathrm{p}$. Des chapitres portent sur " les caractères descriptifs ", « la croissance », « les groupes sanguins ", « les dermatoglyphes » des franco-vietnamiens. «Préambule», p. 3-4.

36. Témoignage dans le documentaire Inconnu présumé français.

37. Compte rendu de l'assemblée générale de l'APPEL, 28/11/1963; propos de René Péchard dans Jean-Claude DARRIGAUD et Jean-Claude DIDELoT, Les enfants..., p. 75.

38. LECA Charles, « Histoire de la FOEFI au Laos des années 1947 à 1975 », Grain de Riz, 1992.

39. Témoignage de Jean-Claude Didelot, recueilli par l'auteur, 21 septembre et 15 décembre 2009.

40. DIDELOT Jean-Claude, Piété filiale... Documents (CD), documents $n^{\circ} 2$ et 3 du chapitre 3 , lettre non datée.

41. Archives nationales, site de Fontainebleau (ANF), 19960015 ministère de l'Intégration et de la Lutte contre l'exclusion, article n² 21, rapport Riviérez sur l'adoption, 1974, p. 26.

42. ANF, 19960015, article $n^{\circ} 22$, statistiques de la FOEFI (1965-1976).

43. Voir les séquences filmées des visites au CAOM dans les documentaires Inconnu présumé français et Né sous $Z$.

44. Archives du ministère des Affaires étrangères, Mission de l'Adoption Internationale (MAI), n 16, télégrammes de Saigon et du MAE, 2 et 4 avril 1975.

45. MAI, $n^{\circ} 16$, correspondance du 5 avril 1975.

46. MAI, $\mathrm{n}^{\circ}$ 16, télégrammes de Saigon du 6 avril 1975.

47. Le Monde, «L'accueil des réfugiés en France. Les enfants du Babylift » par Jean-Claude GUILLEBAUD, 23 mai 1975.

48. DENÉCHÈRE Yves, «De "l'accueil à vie" à l'opération Babylift : adopter et sauver des enfants pendant la guerre du Viêtnam (1965-1975)", Faire la paix, faire la guerre, CTHS, actes du congrès 2011 à Perpignan, sous presse.

49. Témoignage dans le documentaire Inconnu présumé français.

50. Témoignage dans Grain de riz, $\mathrm{n}^{\circ}$ 44, décembre 2011, p. 7.

51. Grain de riz $n^{\circ} 42$, décembre 2010, p. 7 ; $n^{\circ} 44$.

52. Compte rendu de la cérémonie du 7/08/2010, Grain de riz, n 42, décembre 2010, p. 6.

53. «Assemblée des Eurasiennes à l'abbaye de Saint-Rambert-en-Bugey, 14-17 octobre $2010 »$, Grain de riz n 42, décembre 2010, p. 10-12.

54. Site Internet de l'Amicale : [http://www.aet-dalat.net/].

55. Philippe Rostan, Inconnu présumé français. Cf. ci-dessus note 1.

56. Dossier consacré au film de Philippe Rostan, Grain de riz, n 38, décembre 2009. 
57. Plusieurs témoignages d'enfants d'Eurasiens de la FOEFI dans Grain de riz, $\mathrm{n}^{\circ} 38$, décembre 2009, p. 6-11.

58. Par exemples : «Mon père, une quête " et "À mon père, André Antona ", Grain de riz, $\mathrm{n}^{\circ} 40$, décembre 2010, p. 3-4.

59. POLLET-ROUYER Frédérique, Né sous Z. Cf. ci-dessus note 1.

60. "Lettre aux Eurasiens» de Cécile Grandjean, assistante sociale de la FOEFI et « Réponse de Jacqui Maurice » Grain de riz, n³8, décembre 2009, p. 12.

61. CYRULNIK Boris et SERON Claude (dir.), La résilience ou comment renaitre de sa souffrance, Fabert, coll. Penser le monde de l'enfant, 2003, 248p. CYRULNIK Boris, Autobiographie d'un épouvantail, Paris, Odile Jacob, 2010. 279 p.

62. BARI Nadine, CAMARA Laby, L'enfant de Seno, Paris, L'Harmattan, 2011, 123 p.

63. Le Mouvement Retrouvailles est un organisme sans but lucratif, créé 1983, qui vise à faire respecter les droits des personnes adoptées, des parents biologiques et des adoptants.

64. "Ils se sont retrouvés », Grain de riz n 42, décembre 2010, p. 2 ; "Retrouvailles », témoignage de Jean Weber, $\mathrm{n}^{\circ}$ 44, décembre 2011, p. 18-19.

65. Témoignage dans Grain de riz, $\mathrm{n}^{\circ}$ 41, juin 2010, p. 5.

\section{RÉSUMÉS}

La colonisation française en Indochine a induit la naissance d'enfants métis eurasiens de pères français, souvent militaires. À la fin de la guerre, la Fédération des œuvres de l'enfance française en Indochine, avec le soutien de l'État, a décidé de les «rapatrier » en métropole, leurs mères restant sur place. Des années 1950 au milieu des années 1970, des milliers d'enfants ont été regroupés dans des foyers ou dispersés dans des dizaines d'internats dans le but de les acculturer et de les éduquer. Cette migration contrainte, qui relève clairement de la biopolitique, visait une intégration générationnelle des enfants eurasiens. Elle a donné lieu à des débats sur la fin et les moyens de l'entreprise, d'autres acteurs choisissant d'autres voies. Après la dissolution de la FOEFI, des anciens des foyers ont constitué une association afin de se retrouver dans l'identité commune les unissant. L'avancée dans la vie et leurs descendants les ont poussés à évoquer d'autres souvenirs, plus douloureux : la séparation avec la mère, la quête du père.

French colonialism in Indochina led to the birth of mixed Eurasian children of French fathers, often members of the military. At the end of the war, the Fédération des œuvres de l'enfance française en Indochine (FOEFI) [the Federation of charities for French children in Indochina], with the support of the French government, decided to "repatriate" the children to France, while leaving their mothers behind. From the 1950s to the mid-1970s, thousands of children were placed in foster homes or boarding schools in order to be acculturated and educated. This forced migration, clearly inspired by bio-politics, targeted the generational integration of Eurasian children. It gave rise to debates regarding the ends and means of the undertaking; other actors chose different methods. After the dissolution of the FOEFI, the former foster children formed an 
association based on the common identity uniting them. Their advancing age and their descendants pushed them to evoke other, more painful memories: the separation from their mothers and the quest for their fathers.

INDEX

Keywords : history, childhood, colonization, decolonization, France, Indochina, XXth century, migration, boarding schools, memories

\section{AUTEUR}

\section{YVES DENÉCHÈRE}

Professeur d'histoire contemporaine, université Nantes-Angers-Le Mans, CNRS UMR CERHIO 